\section{AB0515 SPANISH SOCIETY OF RHEUMATOLOGY (SER) RECOMMENDATIONS ON PRIMARY ANTIPHOSPHOLIPID SYNDROME (APS). IN A PATIENT WITH OBSTETRIC APS, WHICH TREATMENTS ARE MORE EFFECTIVE? SYSTEMATIC REVIEW}

H. Sánchez Pérez ${ }^{1}$, M. Nishishinya Aquino ${ }^{2}$, C. Pereda ${ }^{3}$, P. Díaz del Campo Fontecha ${ }^{4}$, M. Martínez Zamora ${ }^{5} .{ }^{1}$ Rheumatology, Hospital Universitario de Canarias, La Laguna; ${ }^{2}$ Programa de doctorado en Ciencias de la Educación y el Deporte de la Facultat de Psicologia, Ciències de l'Educació i de l'Esport Blanquerna, Instituto Traumatológico Quirón, Barcelona; ${ }^{3}$ Rheumatology, Hospital Mediterráneo, Almería; ${ }^{4}$ Unidad de Investigación, Sociedad Española de Reumatología, Madrid; ${ }^{5}$ Servicio de Obstetricia y Ginecología, Hospital Clínic, Barcelona, Spain

Background: Pregnancy complications and poor obstetric prognosis are part of the manifestations of APS. The obstetric APS (O-APS) includes 3 or more early miscarriages, 1 or more intrauterine foetal deaths, prematurity secondary to preeclampsia or placental insufficiency, intrauterine growth retardation and HELLP syndrome among others. There is disagreement among different published studies regarding the need of preconceptional or primary thromboprophylaxis (treating with anticoagulant/antiaggregant drugs in the presence of specific autoantibodies but without previous clinical events) and the most effective and safest drug to use, as well as which treatment should be chosen in the case of secondary thromboprophylaxis (treating in the presence of autoantibodies and recurrent miscarriages or previous obstetric complications).

Objectives: To evaluate the available scientific evidence on which treatments are the most effective and safest in O-APS.

Methods: A systematic review (SR) was performed to evaluate the efficacy and safety of different interventions (Aspirin (ASA), Heparin, Antimalarials, Immunoglobulin IV (IVIG), others) in pregnant women with O-APS. We included SR, randomised clinical trials (RCTs) and comparative cohort studies. Result measures on morbidity (prematurity, low birth weight, need for intensive care, impaired cognitive development, preeclampsia, eclampsia, HELLP, abruptio placentae) and mortality included both the newborn and the pregnant woman. A peer review selection and analysis of the studies was carried out (SP,H; NA, MB).

Results: 788 citations were identified (Medline, EMBASE, CENTRAL. May 2017). We included 17 studies: 5 SR, 5 RCTs and 7 cohort studies. Results are shown on the table 1 below:

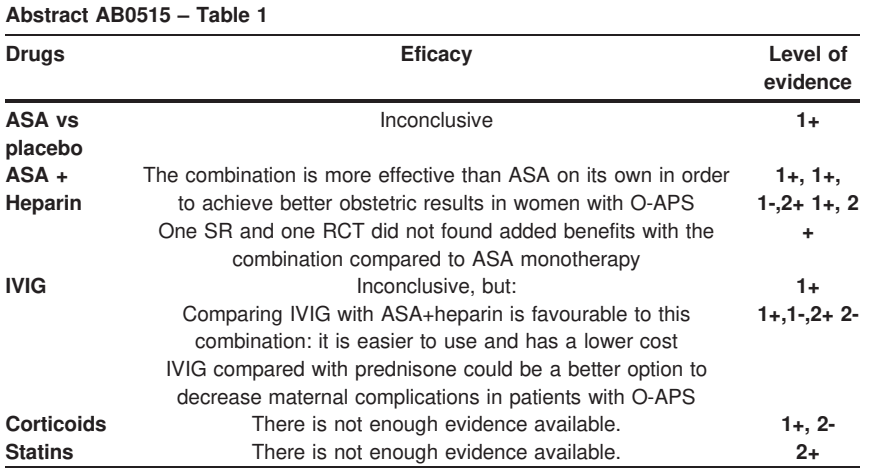

Conclusions: In women with O-APS:

- Secondary thromboprophylaxis: The combination of ASA + Heparin is more effective than ASA monotherapy.

With regards to the use of IVIG, corticosteroids and statins: NO representative conclusions can be drawn from published studies

- Pre-conceptional thromboprophylaxis, primary thromboprophylaxis: NO representative conclusions can be drawn from published studies.

Acknowledgements: This review is part of the preparation of SER Recommendations on Primary APS treatment.

Disclosure of Interest: None declared

DOI: 10.1136/annrheumdis-2018-eular.4842

\section{$\mathrm{AB} 0516$ \\ THERAPEUTIC STRATEGIES AND PROGNOSIS IN CHINESE PATIENTS WITH SEROLOGICALLY ACTIVE CLINICALLY QUIESCENT SYSTEMIC LUPUS ERYTHEMATOSUS}

H. Huang ${ }^{1,2}$, L. Mou ${ }^{1}$, Y. Hao ${ }^{1}$, Z. Zhang ${ }^{1}$, W. Zhou ${ }^{1} .{ }^{1}$ Rheumatology and Clinical Immunology Department, Peking University First Hospital; ${ }^{2}$ Rheumatology and Clinical Immunology Department, First Hospital of Tsinghua University, Beijing, China

Background: SACQ patients with SLE appears to account for $6 \%-12 \%$ of all patients with SLE, but there is disagreement about whether such patients are indeed clinically stable, especially in Chinese patients. And there is no conclusion as to what kind of treatment should be taken for such patients.

Objectives: The aim of our study was to identify the frequency and outcome of SACQ patients with SLE. And we tried to find out potential predictors of flare.

Methods: 682 patients with systemic lupus erythematosus who were followed up for more than 6 months at Peking University First Hospital from January 2007 to December 2015 were summarised. SACQ was defined as an at least a 6 month period with persistent serologic activity and without clinical activity and could be taking a daily dose of prednisone or equivalent less than $7.5 \mathrm{mg}$. Serologically quiescent clinically quiescent (SQCQ) patients and serologically active clinically active (SACA) patients served as control groups. Data including demographics, initial symptoms, duration to $S A C Q$, treatments before and after $S A C Q$, and characteristics of the flare group were analysed.

Results: Of the 682 patients, 170 were SACQ patients (24.9\%), 187 were SQCQ patients, and 325 were SACA patients $(47.7 \%)$. SQCQ patients $(38.61 \pm 15.08$ years old) were older at study start than did SACQ patients ( $38.61 \pm 15.08$ years vs. $32.09 \pm 14.35$ years, $p=0.000$ ), but there was no significant difference between that of SACQ and SACA patients. 56 of the 170 SACQ patients (32.9\%) experienced flare. Corticosteroids (OR $1.323,95 \% \mathrm{Cl} 1.135$ to $1.542 ; \mathrm{p}=0.000$ ) was an independent risk factor for flare, while antimalarials (OR $0.040,95 \% \mathrm{Cl} 0.004$ to $0.418 ; \mathrm{p}=0.007$ ) and immunosuppressants (OR $0.321,95 \% \mathrm{Cl} 0.153$ to 0.673 ; $\mathrm{p}=0.003$ ) were protective factors.

Conclusions: SLE patients with SACQ remained relatively stable, with $32.9 \%$ of patients relapsed. The group of flare patients took greater use of corticosteroids than non-flare ones, whereas antimalarials and immunosuppressants agents were protective factors.

\section{REFERENCES:}

[1] Gladman DD, Urowitz MB, Keystone EC. Serologically active clinically quiescent systemic lupus erythematosus: a discordance between clinical and serologic features. Am J Med 1979;66:210-5.

[2] Huang WN, Tso TK, Wu HC, Yang HF, Tsay GJ. Impaired phagocytosis of apoptotic cell material in serologically active clinically quiescent patients with systemic lupus erythematosis. Int J Rheum Dis 2016;19:1310-6.

[3] Steiman AJ, Gladman DD, Ibañez D, Urowitz MB. Prolonged serologically active clinically quiescent systemic lupus erythematosus: frequency and outcome. J Rheumatol 2010;37:1822-7.

Disclosure of Interest: None declared

DOI: 10.1136/annrheumdis-2018-eular.4624

\section{AB0517 INVASIVE ASPERGILLOSIS IN PATIENTS WITH SYSTEMIC LUPUS ERYTHEMATOSUS: A RETROSPECTIVE STUDY FOCUS ON CLINICAL CHARACTERISTICS AND RISK FACTORS OF IN- HOSPITAL MORTALITY}

H. Mingli ${ }^{1}$, C.-Y. Tsai ${ }^{2} .{ }^{1}$ Rheumatology/Immunology/Allergy, Taipei Veterans General Hospital; ${ }^{2}$ Rheumatology/Immunology/Allergy, Taipei Veterans General Hospital, Taipei, Taiwan, Province of China

Objectives: To analyse the clinical features and outcomes of patients with invasive aspergillosis $(\mathrm{IA})$, the mortality risk factors and all-cause mortality in patients with systemiclupus erythematosus (SLE) in single centre of Taiwan.

Methods: A retrospective study was performed to identify the mortality risk factors associated with IA in patients with systemic lupuserythematosus (SLE). We reviewed the medical records of patients with SLE who were diagnosed with invasive aspergillosis between Jan. 2006 and Jun. 2017 fromTaipei Veterans General Hospital in Taiwan. Clinical and laboratory parameters as well as treatment outcomes were analysed.

Results: Twenty-one patientsdiagnosed with proven $(n=4 ; 19.04 \%)$ and probable $(n=17 ; 80.95 \%)$ invasive aspergillosis according to revised definition by European Organisation for Research and Treatment of Cancer/Invasive Fungal Infections Cooperative Group and the National Institute of Allergy and Infectious Diseases Mycoses Study Group (EORTC/MSG) Consensus Groupwere enrolled in the study and separated into survivors $(n=7 ; 33.33 \%)$ and non-survivors $(n=14$; 
$66.67 \%$ ) groups. All patients in invasive aspergillosis demonstrated significantly high incidence of pneumonia( $n=19 ; 90.47 \%)$, concurrent infections $(n=17$; $80.95 \%)$, and high mortality $(n=14 ; 66.67 \%)$. The coinfections with bacteremia ( $n=8 ; 38.10 \%)$, cytomegalovirus $(n=9 ; 42.86 \%)$, and candida $(n=6 ; 28.57 \%)$ were common in patients with invasive aspergillosis. Gram-negative bacilli (38 isolates; $69.09 \%$ ), of which the most common isolates were carbapenem-resistant Acinetobacter baumannii ( 8 isolates; $21.05 \%$ ). The most common Gram-positive isolates were methicillin-resistant Staphylococcus aureus (6 isolates; $35.29 \%$ ), followed by Vancomycin-resistant Enterococcus (4 isolates; $23.52 \%$ ). The daily glucocorticoid dose $\geq 20 \mathrm{mg}(\mathrm{p}=0.007, \mathrm{HR} 2.000,95 \% \mathrm{Cl} 1.185-3.377)$, rates of recent pulse glucocorticoid therapy $(\mathrm{p}=0.022$, HR $2.800,95 \% \mathrm{Cl} 1.387-5.654)$, rates of azathioprine use $(p=0.022, \mathrm{HR} 2.000,95 \% \mathrm{Cl} 1.185-3.377)$, rates of rituximab within six months ( $p=0.022$, HR $2.000,95 \% \mathrm{Cl} 1.185-3.377$ ), plasmapheresis during hospital course $(p=0.022, \mathrm{HR} 2.000,95 \% \mathrm{Cl} 1.185-3.377)$, and acute respiratory distress syndrome $(p=0.022$, HR 2.000, 95\% Cl 1.185-3.377) among non-survivor group were statistically higher than the survivor group. Non-survivors had significantly higher rate ofconcurrent infections ( $p=0.002$, HR 5.667, 95\% Cl 2.029$15.824)$ and cytomegalovirus (CMV) viremia ( $p=0.040$, HR $1.750,95 \% \mathrm{Cl} 1.112-$ $2.775)$ than survivors. The sensitivity of Galactomannan assay was not good to excludethe diagnosis of invasive aspergillosis. Septic shock $(n=7,50 \%$ of nonsurvivor group) is the most common cause of in-hospital mortality in patients with invasive aspergillosis.

Conclusions: The daily glucocorticoid dose $\geq 20 \mathrm{mg}$, recent pulse glucocorticoid therapy, azathioprine, rituximab within six months, concurrent infections, and CMV viremia are risk factors of in-hospital mortality in invasive aspergillosis patients with Systemic Lupus Erythematosus.

within six months, concurrent infections, and CMV viremia are risk factors of inhospital mortality in invasive aspergillosis patients with Systemic Lupus Erythematosus. within six months, concurrent infections, and CMV viremia are risk factors of in-hospital mortality in invasive aspergillosis patients with Systemic Lupus Erythematosus.

\section{REFERENCE:}

[1] Patterson TF, Thompson GR, Denning DW, et al. Practice Guidelines for the Diagnosis and Management of Aspergillosis: 2016 Update by the Infectious Diseases Society of America. Clinical Infectious Diseases: An Official Publication of the Infectious Diseases Society of America 2016;63 (4):e1-e60. doi:10.1093/cid/ciw326

Disclosure of Interest: None declared

DOI: 10.1136/annrheumdis-2018-eular.1080

\section{$\mathrm{AB} 0518$ \\ EFFICACY OF BELIMUMAB FOR PRIMARY SJÖGREN SYNDROME: RESULTS OF A SYSTEMATIC REVIEW OF THE LITERATURE}

H.S. Park ${ }^{1}$, N. Alvarez-Rivas ${ }^{2}$, P. Diaz del Campo ${ }^{3}$, M. Fernandez-Castro ${ }^{4}$ H. Corominas ${ }^{1}$, J.L. Andreu ${ }^{5}$, V. Navarro-Compán ${ }^{6}$. ${ }^{1}$ Rheumatology, H.U. Sant Pau, Barcelona; ${ }^{2}$ Rheumatology, H.U. Lucus Augustí, Lugo; ${ }^{3}$ Research Unit, SER; ${ }^{4}$ Rheumatology, H.U. Infanta Sofía; ${ }^{5}$ Rheumatology, H.U. Puerta del Hierro Majadahonda; ${ }^{6}$ Rheumatology, H.U. La Paz. IDIPAZ, Madrid, Spain

Background: Belimumab is a human monoclonal antibody that inhibits B-cell activating factor shown to be efficacious in systemic lupus erythematosus. In other B cell mediated autoinmune disease such as primary Sjögren's syndrome(pSS) the efficacy is unclear.

Objectives: To evaluate the efficacy of belimumab in patients with pSS.

Methods: A systematic literature review was perfomed (EMBASE, MEDLINE and Cochrane) as part of the Spanish Rheumatology Society's Recommendations for the Use of Biological Therapies in Primary Sjögren's Syndrome. Inclusion criteria was defined as: Population: patients with pSS according to American-European Consensus Criteria 2002 Intervention: belimumab; Control: synthetic or biologic DMARDs, corticosteroids, ursodesoxicolic acid or placebo; Outcome: efficacy in dryness, glandular and extraglandular manifestations. Studies with $<10$ patients or $<3$ months of follow up were excluded. Two reviewers independently selected the articles and evaluated the quality of the evidence following SIGN guidelines. Results: 3 articles were included out of 135 . All of them published results from the same study ${ }^{1}$ at different timepoints. The study design was experimental but with a small sample size and no control group or randomization.

The article of Mariette et al $2015^{1}$ evaluated the efficacy and safety of belimumab in 30 patients with systemic activity or early disease until week 28(W28). There was a significant decrease in mean ESSDAI (8.8 to $6.3 \mathrm{p}=0.02)$, ESSPRI (6.4 to $5.6 \mathrm{p}=0.02$ ) and VAS for dryness (7.8 to $6.2 \mathrm{p}=0.02$ ). Physician's VAS for disease systemic activity also decreased in $43 \%$ as well as parotid inflammation in $76.9 \%$ of patients. Also, B cell biomarkers decreased: IgG (21.2 to $18.2 \mathrm{~g} / \mathrm{L}, \mathrm{p}=0.02), \lg \mathrm{A}$ (3.7 to $3.3 \mathrm{~g} / \mathrm{L}, \mathrm{p}=0.04$ ), $\operatorname{lgM}$ ( 1.6 to $1.3 \mathrm{~g} / \mathrm{L}, \mathrm{p}<0.001)$, RF (146.2 to $106.7 \mathrm{UI}$ $\mathrm{p}<0.001)$ and number of $B$ cells $(187.2$ a $65.1 / \mathrm{mm}, \mathrm{p}<0.001)$.
The study of De Vita et al $2015^{2}$ compared results at week 52 (W52) and W28 in 19 patients, of whom 15 had previously responded to treatment. Of these 15 patients 13 maintained response and 3 out of the 4 patients that did not respond achieved primary outcome. The improvement at W52 continued for ESSDAI, glandular inflammation, lympadenopathy and joints. B cell biomarkers remained stable. Overall items conributing to ESSDAI descreased but only physician's VAS for disease systemic activity was statistically significant (3.2 W28 vs 2.5 W52; $p=0.04)$.
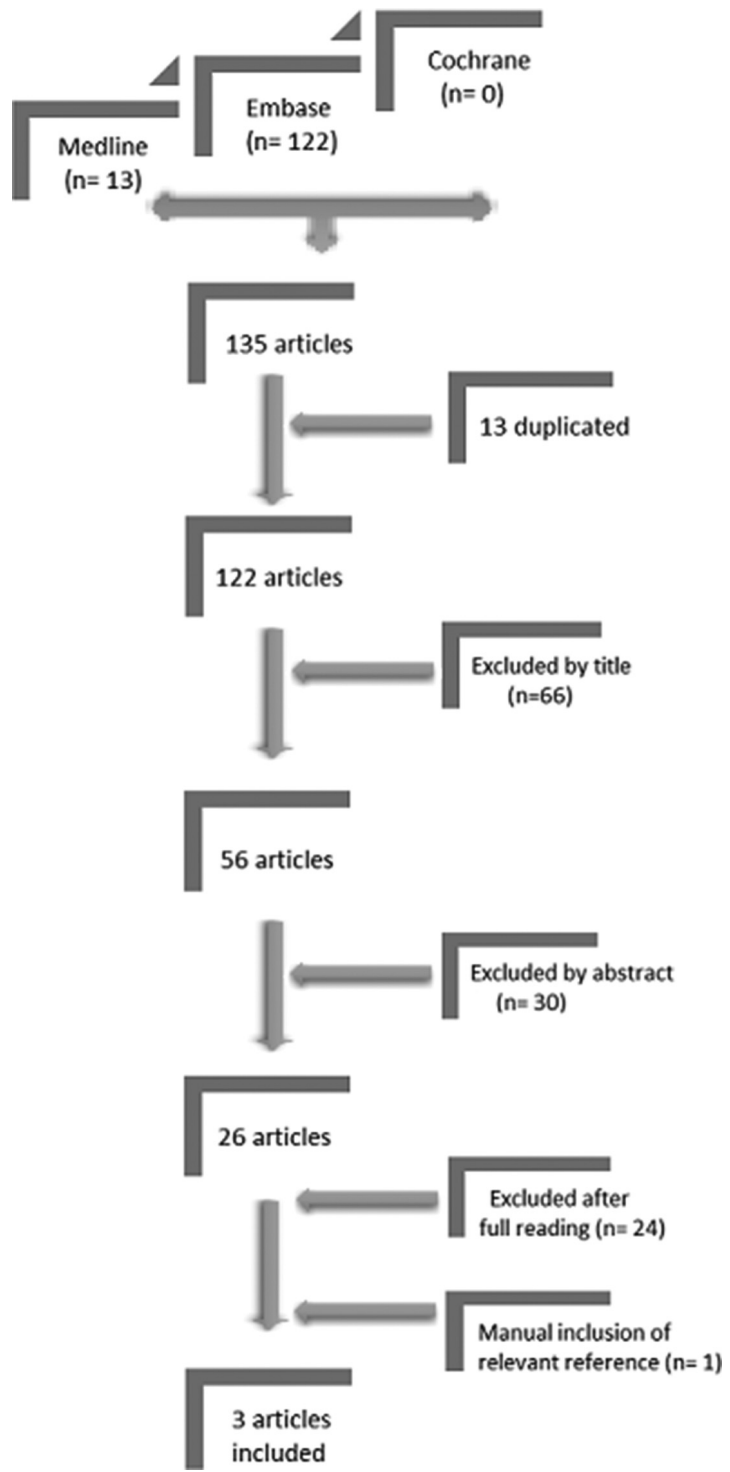

Abstract AB0518 - Figure 1

The study of Quartuccio et al. ${ }^{3}$ compared clinical and lab variables of W52 with 12 months of follow up after interrupting belimumab. A significant decrease in ESSDAl in 9 out of 13 patients $(3.5 \pm 3.7$ vs $7.0 \pm 5.7 ; p<0.01)$ was observed as well as in RF (52 vs 69Ul; $p<0.01$ ), IgM (131.9 vs $165 \mathrm{mg} / \mathrm{dl} ; \mathrm{p}=0.04)$ and BLyS (1304 vs $2882 \mathrm{pg} / \mathrm{ml} ; \mathrm{p}=0.01$ ).

Conclusions: Published evidence to determine the efficacy of belimumab in primary Sjögren is limited and poor. Belimumab seems to be effective to reduce systemic activity, parotid enlargement, dryness, lymphadenopathies, articular manifestation, fatigue and $\mathrm{B}$ cell biomarkers.

\section{REFERENCES :}

[1] ARD 2015;74:526-531.

[2] Rheumatology 2015;54(12):2249-56.

[3] Clin Exp Rheumatol 2016;34(2):311-4.

Acknowledgements: This Project has been financed by the SER. Disclosure of Interest: None declared DOI: 10.1136/annrheumdis-2018-eular.2938 